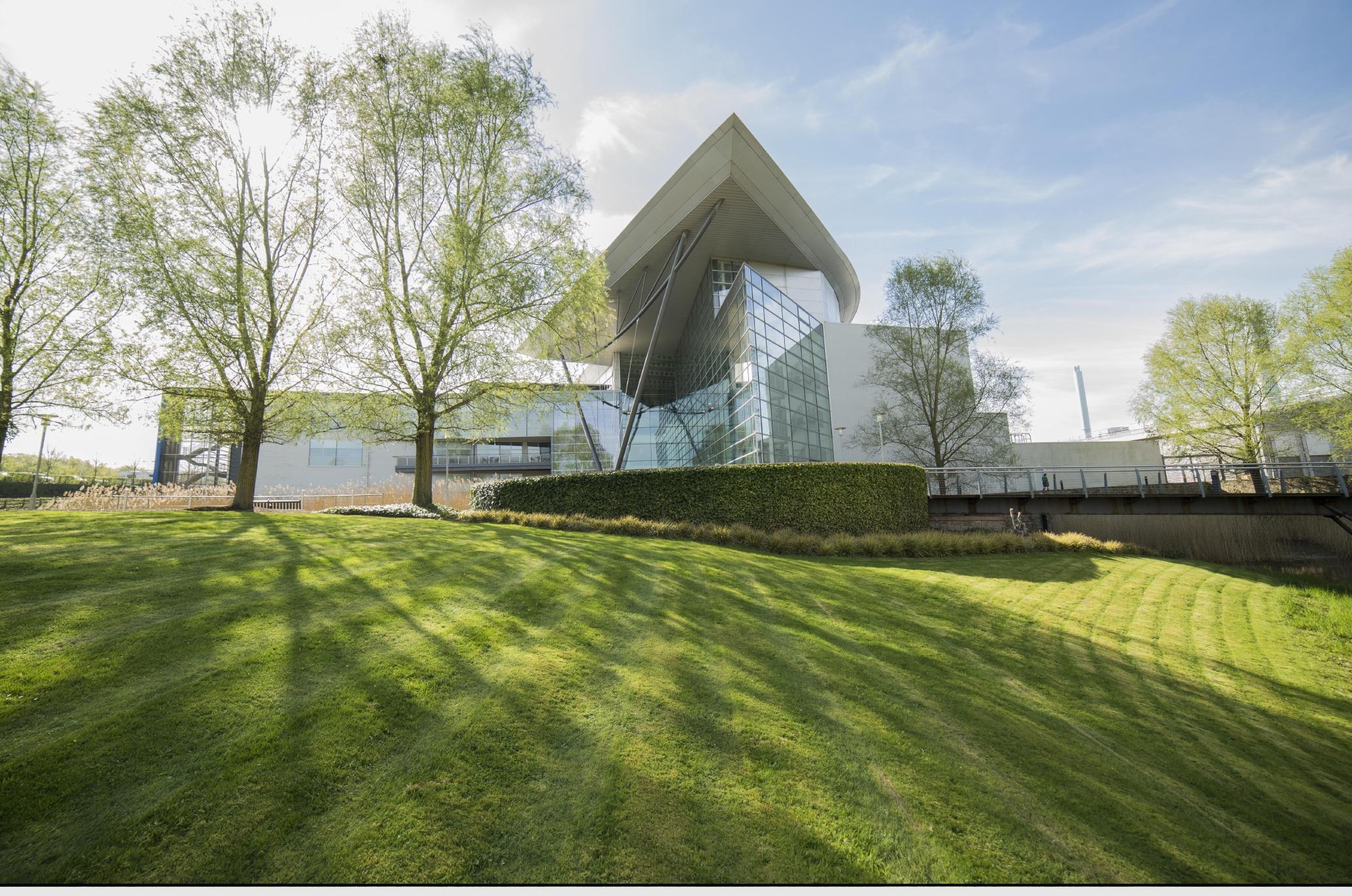

\title{
EI SPC de UK Met Office MOGREPS
}

KEN MYLNE

$U K M O$

The Met Office Global and Regional Ensemble Prediction System (MOGREPS) is an ensemble system that produces uncertainty information, primarily for short-range forecasts. MOGREPS is primarily designed to aid the forecasting of rapid storm development, wind, rain, snow and fog.

https: //www. metoffice. gov. uk/research/weather/ensemble-forecasting/mogreps Met OfFICE, United Kingdom

El Servicio Meteorológico británico, United Kingdom Met Office o, abreviadamente, Met Office disfruta, entre otros sistemas de predicción numérica, de un sistema de predicción por conjuntos (SPC) que abarca todas las escalas tanto espaciales como, por ende, temporales. El así llamado Met Office Global and Regional Ensemble Prediction System, MOGREPS, es decir, SPC global y regional de Met Office, consta de una componente global y otra regional para generar información sobre la incertidumbre atmosférica, principalmente enfocado a las previsiones a corto plazo. El sistema está diseñado especialmente para ayudar en la predicción del desarrollo de tormentas rápidas, viento, lluvia, nieve y niebla.

Palabras clave: sistema de predicción por conjuntos MOGREPS de UKMO, MOGREPS-G, MOGREPS-UK. 


\subsection{Introducción y breve historia}

El sistema de predicción por conjuntos global y regional del Servicio Meteorológico británico, en inglés Met Office Global and Regional Ensemble Prediction System, MOGREPS, está construido con base en el modelo de predicción numérica unificado correspondiente, Met Office Unified Model, UM. MOGREPS se implementó primeramente en 2005 y se diseñó desde el principio como un SPC regional para predicciones a corto plazo, pero incluyendo un SPC global (MOGREPS-G) que proporcionase condiciones de contorno y perturbaciones consistentes para el SPC regional. Una versión de alcance 15 días del global llamada MOGREPS-15 se estuvo integrando desde 2006 hasta 2014 como contribución de UKMO al proyecto y archivo TIGGE [18] (cap. 26 en la página 387), un proyecto de investigación del programa The Observing System Research and Predictability Experiment (THORPEX) de SPC multimodelo en el que se hacían disponibles las predicciones para fines de investigación. En 2014 MOGREPS-G se amplió de resolución hasta los primeros 7 días y pasó a ser un SPC global operativo, sustituyendo a MOGREPS-15 en el archivo TIGGE [18] y en las correspondientes predicciones multimodelo.

Cuando se implementó MOGREPS por primera vez en 2005 [4], la componente regional MOGREPS-R era un SPC de 24 miembros con una malla de $24 \mathrm{~km}$ de resolución abarcando el Atlántico Norte y Europa, con un beneficio de resolución significativo en comparación con los SPC globales disponibles en la época. MOGREPS-G se implementó con Ensemble Transform Kalman Filter (ETKF [2] sec. 16.2.2 en la página 246) para proporcionar perturbaciones en las condiciones iniciales que se añadían al análisis global de Met Office. Se aplicaban perturbaciones físicas estocásticas usando un esquema de Parámetros Aleatorios y, algo más tarde, se introdujo [5] un esquema llamado Stochastic Kinetic Energy Backscatter, SKEB [1]. MOGREPS-R se implementó inicialmente como una reducción de escala o downscaling (sec. 10.1.3 en la página 132) del análisis global y sus perturbaciones. Más tarde MOGREPS-R se mejoró para usar las perturbaciones globales ETKF centradas en el análisis regional de alta resolución. Durante un tiempo se implementó [5] un ETKF regional, pero tuvo peor rendimiento que el ETKF global, de modo que se reemplazó. Se introdujeron posteriormente mejoras con un esquema de inflación adaptativo [3], primero con el uso de observaciones de satélite para aumentar la estabilidad del esquema [6] y más tarde permitiendo la variación vertical del factor de inflación [7].

Incluso en los primeros planes, uno de los objetivos era prepararse para proporcionar condiciones de contorno laterales para un futuro SPC de escala convectiva (convection-permitting). Se reconocía así que cuando los modelos de escala convectiva fuesen plausibles con el avance en supercomputación, la aproximación SPC sería esencial para tratar con los errores de rápido crecimiento en esas escalas (sec. $22.1 \mathrm{en}$ la página 334). El primer SPC de escala convectiva, MOGREPS-UK [8], se implementó en 2012 como parte de un proyecto de demostración de predicción (forecast demonstration project) asociado a los Juegos Olímpicos de Londres 2012. Usaba un modelo de resolución variable, con malla de $2.2 \mathrm{~km}$ en el interior, estirándose hasta $4 \mathrm{~km}$ en los bordes. De este modo podía anidarse (ver anidamiento) directamente en MOGREPS-G, que en ese momento había mejorado con una malla de $33 \mathrm{~km}$, haciendo innecesario el intermedio de MOGREPS-R, que se retiró en esta etapa. MOGREPS-G se modificó para integrarse cuatro veces al día con 12 miembros para proporcionar condiciones de contorno actualizadas a un MOGREPSUK de 12 miembros cada seis horas. Combinando las dos últimas pasadas de MOGREPS-G con la técnica time-lagged ensemble [12] (sec. 13.5 en la página 174) se generaban productos con 24 miembros. MOGREPS-UK se implementó inicialmente como un SPC de reducción de escala usando condiciones iniciales y perturbaciones de MOGREPS-G. Más tarde, en 2016, se mejoró para usar las perturbaciones globales centradas en el análisis sobre UK de escala convectiva. 


\begin{tabular}{lll}
\hline SPC & MOGREPS-G & MOGREPS-UK \\
\hline Tipo & Mono-modelo con perturbaciones & Mono-modelo con perturbaciones \\
\hline Modelo base & Unified Model (UM) & Unified Model (UM) \\
\hline Tamaño & 18 miembros & 12 miembros \\
\hline Pasadas & 4 diarias: 00, 06, 12, 18 UTC & 4 diarias: 00, 06, 12, 18 UTC \\
\hline Alcances & Hasta 7 días & Hasta 54 h \\
\hline Resolución horizontal & $20 \mathrm{~km}$ & Variable, 2.2 km en el interior \\
\hline Dominio & Global & UK \\
\hline Perturbaciones en las & $\begin{array}{l}\text { LETKF [10] basado en un SPC de 44 } \\
\text { miembros que se integra sólo en el ciclo } \\
\text { condiciones iniciales }\end{array}$ & $\begin{array}{l}\text { Reducción de escala (downscaling) directa } \\
\text { de MOGREPS-G. }\end{array}$ \\
\cline { 2 - 3 } & $\begin{array}{l}\text { Se anilación } \\
\text { den al ańlis }\end{array}$ &
\end{tabular}

Se añaden al análisis global 4DVAR que usa el ensemble para estimar los errores del campo previo dependientes del flujo

Las perturbaciones se centran en el análisis 4D-VAR de UK, ciclos cada hora.

Esquema de Parámetros Aleatorios que Esquema de Parámetros Aleatorios que modifica estocásticamente los esquemas modifica estocásticamente los esquemas

Perturbaciones en el modelo de las parametrizaciones físicas de las parametrizaciones físicas

Stochastic Kinetic Energy Backscatter (SKEB) [19]

Perturbaciones en temperatura de la superficie del mar, humedad del suelo y temperatura del suelo profundo [20]

Perturbaciones estocásticas en la Planetary Boundary Layer (PBL) para temperatura y humedad

Se generan productos probabilistas con un Combinaciones de las dos últimas pasadas

Posproceso con métodos de vecindario para generar productos con 36 miembros usando la técnica time-lagged ensemble [12] método de vecindario incorporando vecinos de las celdas cercanas para tener en cuenta la incertidumbre espacial no abarcada por el SPC de 12 miembros (Figura 23.2 en la página 365).

Tabla 23.1: Tabla resumen de características de los SPC MOGREPS-G, global y MOGREPS-UK, regional.

\subsection{MOGREPS en 2017}

En 2017 MOGREPS consta de un SPC global, MOGREPS-G, a $20 \mathrm{~km}$ de resolución para predicciones de hasta una semana y un SPC regional, MOGREPS-UK, a $2.2 \mathrm{~km}$ para predicciones de corto plazo para el Reino Unido. Pueden encontrarse más detalles, así como referencias, en el sitio web de UK MetOffice https://www.metoffice.gov.uk/research/ weather/ensemble-forecasting/mogreps. Las características de ambos SPC quedan sintetizadas en la Tabla 23.1.

El posproceso que se realiza de MOGREPS-G proporciona a los predictores una amplia variedad de productos, incluyendo sellos (sec. 27.2.1 en la página 402) y mapas de probabilidad (sec. 27.6.1 en la página 416) para un rango amplio de umbrales. El sistema identifica ciclones extratropicales y sistemas frontales y se dibujan como características sinópticas [9]. Un servicio de mapas web (wms) proporciona un mapa global de peligros. 

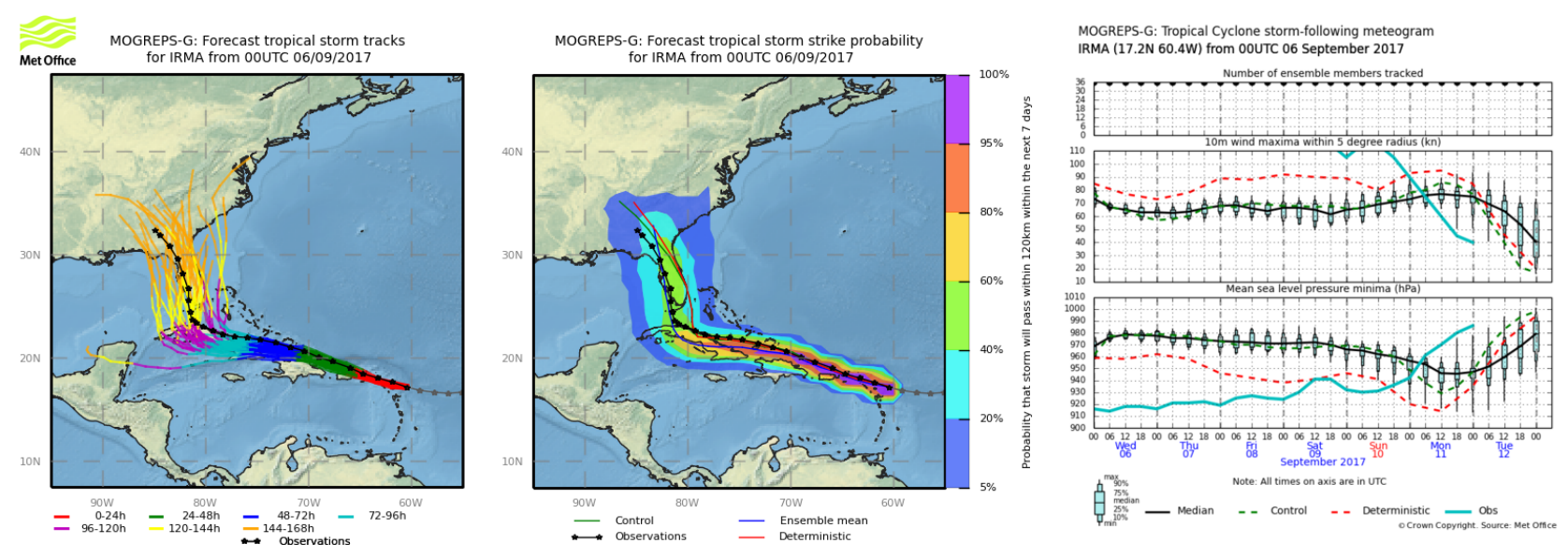

Figura 23.1: Predicciones de MOGREPS-G para el huracán IRMA, pasada de 00 UTC de 06-09-2017. Izquierda: trayectorias SPC. Centro: probabilidades de impacto. Derecha: EPSgramas de intensidad de la tormenta. Las líneas negras punteadas indican la trayectoria observada en las imágenes izquierda y central, mientras que la línea color cían muestra la intensidad observada (figura cortesía de HELEN TITLEY, UKMO).

El citado mapa de peligros se genera combinando predicciones probabilistas de MOGREPS-G y ECENS (sec. 19.3 en la página 293) para dar un resumen mundial del tiempo potencial de alto impacto, incluyendo probabilidades de fenómenos extremos muy apartados de valores medios climatológicos (aquellos que excedan el percentil 99 de la climatología local) [17].

Los ciclones tropicales se rastrean antes y después de su formación, poniendo productos a disposición de los Centros Meteorológicos Regionales Especializados (Figura 23.1). Para las predicciones a unos cuantos días vista los miembros del SPC se agrupan mediante un sistema de regímenes de tiempo atmosférico para resumir las probabilidades de tipos de tiempo y los potenciales de tiempo de impacto en UK [14]. Se generan a cinco días vista para UK primeras conjeturas (first-guess) de avisos de fenómenos adversos basadas en el posible riesgo [13].

La Tabla 23.1 en la página anterior destaca las características esenciales del SPC MOGREPS-UK. Pueden encontrarse todos los detalles de forma más completa en [8]. MOGREPS-UK se utiliza como condiciones iniciales y de contorno (en inglés se utiliza el teŕmino forzar) para una serie de modelos de impacto meteorológico que dan soporte a toma de decisiones basada en riesgo de tiempo de alto impacto. Entre estos modelos podemos citar los de avisos de primera conjetura (first-guess) de fenómenos adversos para UK, un modelo de peligro de volcado de vehículos que identifica el aumento del riesgo para el transporte por carrete- ra debido a fuertes vientos y, por último, riesgos de inundaciones por desbordamiento de ríos o por lluvias torrenciales.

\subsection{Planes futuros}

Los planes de mejora de MOGREPS para los próximos años a partir de 2017 intentarán afinar la dispersión generada para ser más consistente con el ritmo de crecimiento de los errores, es decir, afinar la relación dispersión-error (sec. 15.8.2 en la página 223). Los planes inmediatos se centran en MOGREPS-UK para que aproveche la potencia de la reciente mejora del superordenador de alto rendimiento Cray XC40 (cap. 11 en la página 145). El MOGREPS-UK se reestructurará para explotar el análisis 4D-VAR de escala convectiva en UK con ciclos cada hora, integrando 3 miembros cada hora centrados en el último análisis y generando así un SPC con desfase de tiempo (timelagged, sec. 13.5 en la página 174) de 18 miembros combinando los seis últimos ciclos horarios. Así se introducen, por primera vez, perturbaciones de escala convectiva en las condiciones iniciales mediante el uso de múltiples análisis, reduciendo así la tendencia del SPC a seguir la última predicción determinista. Experimentalmente, las predicciones se extenderán hasta 5 días para probar el beneficio de la reducción de escala a la escala convectiva en el medio plazo temprano, particularmente para acumulaciones de precipitación provocadoras de inundaciones. 


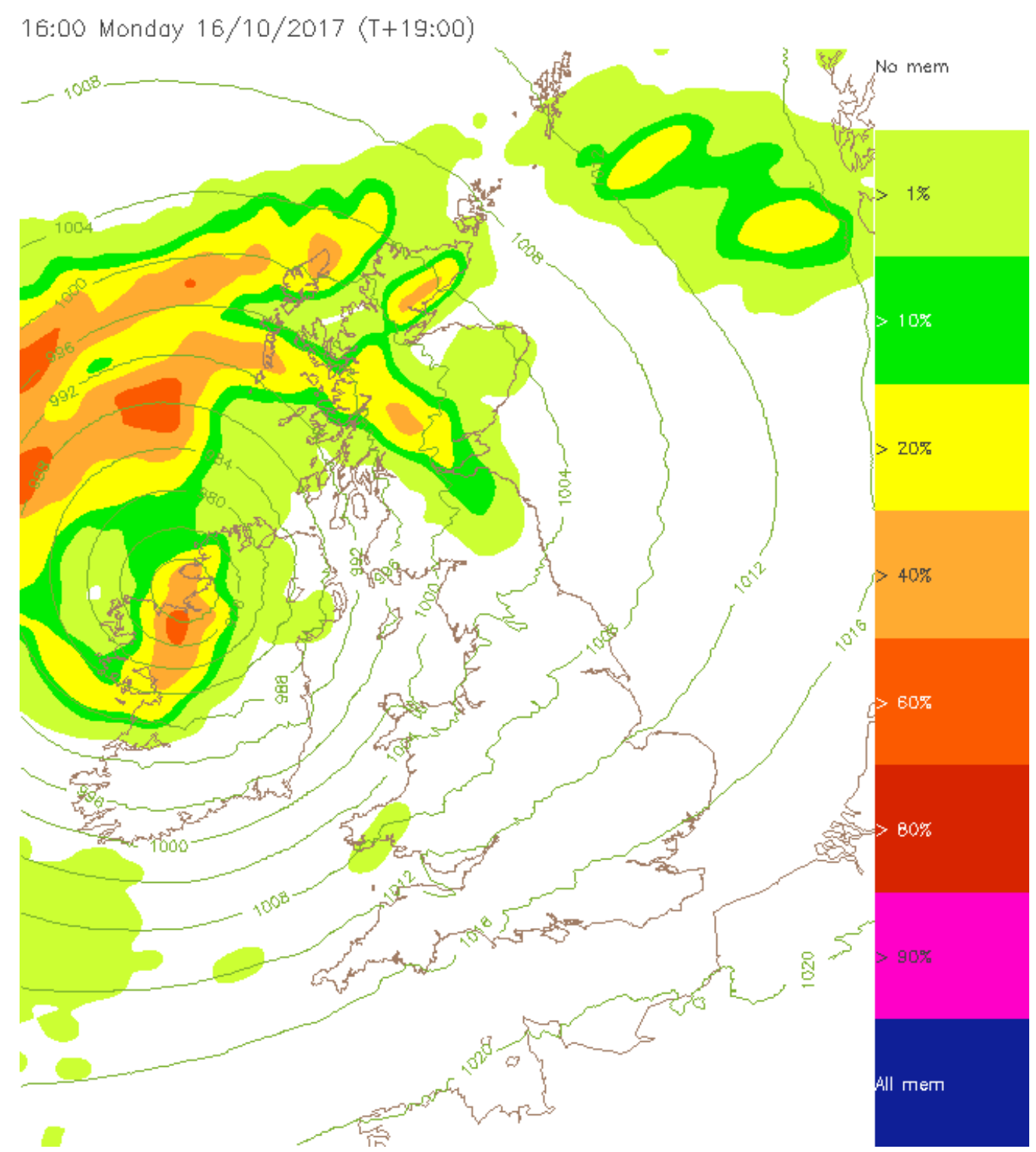

Figura 23.2: Ejemplo de mapa de probabilidad, procesado con método de vecindario, de intensidad de precipitación superior a $4 \mathrm{~mm} / \mathrm{h}$ a partir de MOGREPS-UK.

Un reemplazo primordial para los sistemas de posproceso está también en fase de desarrollo para explotar los ciclos horarios de MOGREPS-UK, mezclados con otros sistemas de predicción numérica, en un marco plenamente probabilista.

Otros planes incluyen:

- Reemplazar ETKF por un sistema de asimilación por conjuntos (EDA [11], sec. 16.2.5 en la página 248) basado en 4D-Ensemble-VAR para mejorar la dispersión de las perturbaciones iniciales de MOGREPS-G.
- Sistema perfeccionado de perturbaciones estocásticas en MOGREPS-G mediante una formulación actualizada de SKEB, reemplazo del método de $\mathrm{Pa}-$ rámetros Aleatorios por SPPT [15] (sec. 17.4.4 en la página 269) y la introducción de un término de forzamiento estocástico basado en incrementos del análisis seleccionados aleatoriamente [16].

- Perturbaciones en las parametrizaciones de superficie en MOGREPS-UK.

- Sistema de asimilación por conjuntos (EDA) en UK para generar perturbaciones de escala convectiva para MOGREPS-UK. 


\subsection{Referencias}

[1] Berner, J. y col. "A Spectral Stochastic Kinetic Energy Backscatter Scheme and Its Impact on Flow-Dependent Predictability in the ECMWF Ensemble Prediction System". En: Journal of the Atmospheric Sciences 66.3 (mar. de 2009), páginas 603-626. ISSN: 00224928. DOI: $10.1175 / 2008$ JAS2677 1 (citado en página 362 ).

[2] Bishop, Craig H., Etherton, Brian J y MAJUmDAR, Sharanya J. "Adaptive sampling with the ensemble transform Kalman filter. Part I: Theoretical aspects". En: Monthly weather review 129.3 (2001), páginas 420-436 (citado en página 362).

[3] Bocquet, Marc y Sakov, Pavel. "An iterative ensemble Kalman smoother". En: Quarterly Journal of the Royal Meteorological Society 140.682 (2014), páginas 1521-1535. DOI: 10.1002 / qj . 2236 (citado en página 362).

[4] BOWLER, Neill E. y col. "The MOGREPS short-range ensemble prediction system". En: Quarterly Journal of the Royal Meteorological Society 134.632 (abr. de 2008), páginas 703-722. ISSN: 00359009. DOI: 10 . 1002/qj . 234 (citado en página 362).

[5] BOWLER, Neill E y col. "The local ETKF and SKEB: Upgrades to the MOGREPS short-range ensemble prediction system". En: Quarterly Journal of the Royal Meteorological Society 135.640 (2009), páginas 767-776. DOI: 10 .1002/qj . 394 (citado en página 362 ).

[6] FLOWERDEw, Jonathan y BowLer, Neill E. "Improving the use of observations to calibrate ensemble spread". En: Quarterly Journal of the Royal Meteorological Society 137.655 (2011), páginas 467-482. DOI: 10 .1002/qj . 744 (citado en página 362).

[7] Flowerdew, Jonathan y Bowler, Neill E. "On-line calibration of the vertical distribution of ensemble spread". En: Quarterly Journal of the Royal Meteorological Society
139.676 (2013), páginas 1863-1874. DOI: 10 . 1002/qj . 2072 (citado en página 362).

[8] Hagelin, Susanna y col. "The Met Office convective-scale ensemble, MOGREPSUK". En: Quarterly Journal of the Royal Meteorological Society 143.708 (2017), páginas 2846-2861. DOI: 10.1002/qj . 3135 (citado en páginas 362, 364).

[9] Hewson, Tim D y Titley, Helen A. "Objective identification, typing and tracking of the complete life-cycles of cyclonic features at high spatial resolution". En: $M e-$ teorological Applications 17.3 (2010), páginas 355-381. DOI: 10 . 1002 /met . 204 (citado en página 363).

[10] Hunt, Brian R., Kostelich, Eric J. y SzUnYogh, Istvan. "Efficient data assimilation for spatiotemporal chaos: A local ensemble transform Kalman filter". En: Physica D: Nonlinear Phenomena 230.1-2 (jun. de 2007), páginas 112-126. ISSN: 01672789. DOI: 10 . 1016/J . PHYSD . 2006 . 11. 008 (citado en página 363).

[11] ISAKSEN, Lars y col. Ensemble of data assimilations at ECMWF. European Centre for Medium-Range Weather Forecasts, 2010 (citado en página 365).

[12] Lu, Chungu y col. "Short-Range Numerical Weather Prediction Using Time-Lagged Ensembles". En: Weather and Forecasting 22.3 (jun. de 2007), páginas 580-595. ISSN: 08828156. DOI: 10.1175/WAF999. 1 (citado en páginas 362, 363).

[13] Neal, Robert A y col. "Ensemble based first guess support towards a risk-based severe weather warning service". En: Meteorological Applications 21.3 (2014), páginas 563-577. DOI: 10 . $1002 /$ met . 1377 (citado en página 364).

[14] NEaL, Robert y col. "A flexible approach to defining weather patterns and their application in weather forecasting over Europe". En: Meteorological Applications 23.3 (2016), páginas 389-400. DOI: 10.1002/met. 1563 (citado en página 364). 
[15] PAlmer, T N y col. "Stochastic parametrization and model uncertainty". En: ECMWF Tech. Memo 598 (2009), páginas 1-42 (citado en página 365$)$.

[16] Piccolo, Chiara y Cullen, Mike. "Ensemble data assimilation using a unified representation of model error". En: Monthly Weather Review 144.1 (2016), páginas 213-224 (citado en página 365).

[17] Robbins, C. J. y Titley, H. A. "Evaluating high-impact weather forecasts from the Met Office Global Hazard Map using a global impact database". En: Met. Apps. pendiente (2018) (citado en página 364).

[18] SwINBAnK, Richard y col. "The TIGGE project and its achievements". En: Bulle- tin of the American Meteorological Society 97.1 (2016), páginas 49-67 (citado en página 362).

[19] TENnANT, Warren J y col. "Using a stochastic kinetic energy backscatter scheme to improve MOGREPS probabilistic forecast skill". En: Monthly Weather Review 139.4 (2011), páginas 1190-1206 (citado en página 363).

[20] Tennant, Warren y Beare, Sarah. "New schemes to perturb sea-surface temperature and soil moisture content in MOGREPS". En: Quarterly Journal of the Royal Meteorological Society 140.681 (2014), páginas 1150-1160. DOI: 10 . 1002/qj . 2202 (citado en página 363). 
